Research Article

\title{
Wideband Polarization Conversion Metasurface for RCS Reduction of Antennas
}

\author{
Tao Hong $(\mathbb{D}$, Ke Peng, Yue Gu, Wen Jiang $(\mathbb{D}$, and Shuxi Gong \\ National Key Laboratory of Antennas and Microwave Technology, Xidian University, No. 2 South Taibai Road, Xi'an, China \\ Correspondence should be addressed to Tao Hong; thong@mail.xidian.edu.cn
}

Received 4 June 2018; Revised 19 August 2018; Accepted 30 August 2018; Published 27 September 2018

Academic Editor: Flaminio Ferrara

Copyright (C) 2018 Tao Hong et al. This is an open access article distributed under the Creative Commons Attribution License, which permits unrestricted use, distribution, and reproduction in any medium, provided the original work is properly cited.

\begin{abstract}
Polarization conversion metasurface (PCM) with wide operating band and high polarization conversion ratio is proposed for antenna radar cross-section (RCS) reduction. A 3rd-order quadrate fractal structure (QFS) is designed as the PCM element that the polarization conversion ratio is greater than $80 \%$ from $10.45 \mathrm{GHz}$ to $32.15 \mathrm{GHz}$ (relative bandwidth of $101.9 \%$ ). The PCM is applied in the RCS reduction of microstrip antennas by chessboard-like arrangement on the antenna. The performances of the proposed PCM and antenna are studied by measurement and simulation. The results show that, with the radiation performance hold, the monostatic RCS of the proposed antenna is obviously reduced from $8 \mathrm{GHz}$ to $28 \mathrm{GHz}$ which completely covers the operating band of the antenna.
\end{abstract}

\section{Introduction}

With the rapid development of new system of radar, the stealth characteristics of various military carriers such as aircrafts and warships are getting more and more attention. Low radar cross-section (RCS) property of the carrier can reduce the ability of the enemy's radar to detect the target, thereby improving the battlefield viability and penetration ability of one's own goals. Antennas are essential for a variety of radio equipment, and they are responsible for transmitting and receiving electromagnetic waves. In a complex electromagnetic environment, how to improve the carrier stealth performance while maintaining the normal work of the antenna is a difficult task, which is becoming a hot spot.

The working principle of the checkerboard surfaces is that the scattering field of the adjacent checkerboard blocks is cancelled because the amplitude is the same and the phase difference is $180^{\circ}$ [1-6]. A wider $10 \mathrm{~dB}$ RCS reduction bandwidth of $60 \%$ is obtained by combining two different types of electromagnetic band gap (EBG) structures for the square and hexagonal checkerboard surfaces [1]. Checkerboard surfaces consisting of two different EBG structures can realize $10 \mathrm{~dB}$ RCS reduction bandwidths of $61 \%$ and $24 \%$ [2]. In [5], the RCS is reduced by more than $10 \mathrm{~dB}$ in the band of $9.4 \mathrm{GHz}-23.28 \mathrm{GHz}$. The size adjustment metasurface (SA-MS) is composed of a wideband polarization conversion surface and a partially reflecting surface (PRS), which obtained a wideband RCS reduction in the frequency range of $9 \mathrm{GHz}-17 \mathrm{GHz}$ [6].

In the past few years, some research activities on the RCS reduction of antenna with polarization conversion characteristics have been reported, which is acquired by polarization conversion metasurface (PCM). The split-ring sheet PCM can realize $5 \mathrm{~dB}$ RCS reduction in a wide range from $8.6 \mathrm{GHz}$ to $24.5 \mathrm{GHz}$, and the relative bandwidth reaches up to $96 \%$ [7]. In 2016, Liu et al. loaded $45^{\circ}$ angle symmetric split-ring resonators around a microstrip antenna, achieving RCS reduction in $4.2 \mathrm{GHz}, 6.75 \mathrm{GHz}, 9 \mathrm{GHz}$, and $12 \mathrm{GHz}$ bands [8]. They proposed a new PCM that was placed on the surface of the slot array antenna, reducing the monostatic RCS and preserving the radiation characteristics [9]. The proposed metasurface has a remarkable $(5 \mathrm{~dB})$ monostatic RCS reduction characteristic from $8.6 \mathrm{GHz}$ to $24.5 \mathrm{GHz}$ 


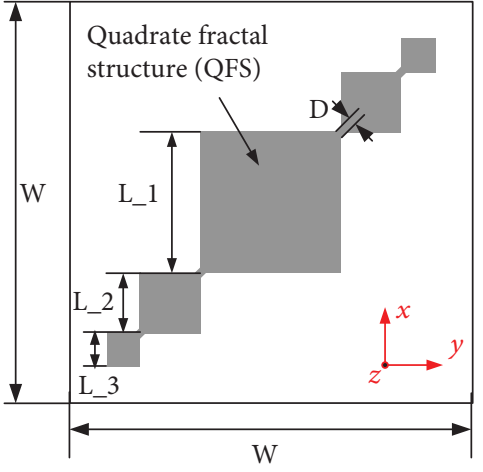

(a)

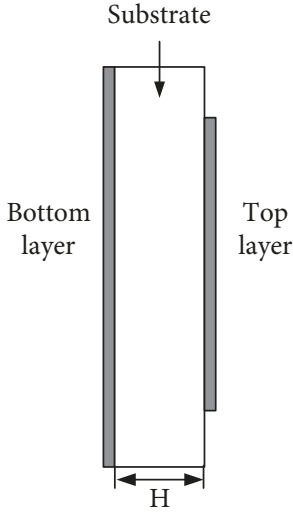

(b)

Figure 1: Geometry of the PCM unit cell: (a) Top view; (b) side view.

[10]. In [11], an ultrawideband dual-patch PRS is presented to widen the polarization rotation bandwidth from $49 \%$ to $97 \%$ with a high polarization conversion ratio (PCR) of $96 \%$, and $10 \mathrm{~dB}$ RCS reduction is achieved over 98\%. A microstrip antenna array with a metasurface based on sizeadjustable unit cells is designed, which is capable of achieving a RCS reduction larger than $5 \mathrm{~dB}$ from $6.2 \mathrm{GHz}$ to $27.3 \mathrm{GHz}$ (126.0\% frequency bandwidth) [12].

In order to improve the bandwidth and polarization conversion efficiency of polarization conversion metasurfaces, novel polarization devices have been proposed in many studies. An ultrathin chiral metamaterial slab stacked with twisted complementary split-ring resonators realized conversion efficiency of up to $96 \%$ covering a bandwidth of $24 \%$ of the central wavelength [13]. Shi et al. realized the polarization rotation modulation of the transmitted waves in three frequency bands of $9.82 \mathrm{GHz}, 11.39 \mathrm{GHz}$, and $13.37 \mathrm{GHz}$ by loading four symmetrical rotating open rings on both sides of the dielectric substrate [14]. Chen et al. used an asymmetric double-aperture resonant ring structure to achieve $80 \%$ reflection-wave polarization rotation modulation in the band from $7 \mathrm{GHz}$ to $20 \mathrm{GHz}$ [15]. The polarization conversion metasurface of the open-elliptical ring resonator is capable of achieving the polarization rotation efficiency of greater than $85 \%$ over a frequency range of $104.5 \%$ of the relative bandwidth [16]. An ultrathin wideband polarization conversion composing of arrays of oval ring pattern obtained a polarization conversion ratio (PCR) of over $68.6 \%$ from $8.0 \mathrm{GHz}$ to $18.0 \mathrm{GHz}$ [17]. An ultrawideband and highperformance polarization converter that makes use of a double V-shaped metasurface is proposed, with a mean PCR of $90 \%$ from $12 \mathrm{GHz}$ to $27.9 \mathrm{GHz}$ [18]. The polarization conversion metasurfaces consist of a dielectric substrate sandwiched between double split-ring resonators (DSRR), disk resonators, and a ground plane, whose polarization conversion ratio is above $94 \%$ for the $\mathrm{Ku}$-band and $90 \%$ $(11.5 \mathrm{GHz}$ to $20 \mathrm{GHz})$ for the Ka-band $(28.8 \mathrm{GHz}$ to $34 \mathrm{GHz})$ [19].

In this paper, an ultrawideband and high-efficiency PCM is proposed. By a novel quadrate fractal structure element, the operating band from $10.45 \mathrm{GHz}$ to $32.15 \mathrm{GHz}$ is achieved.

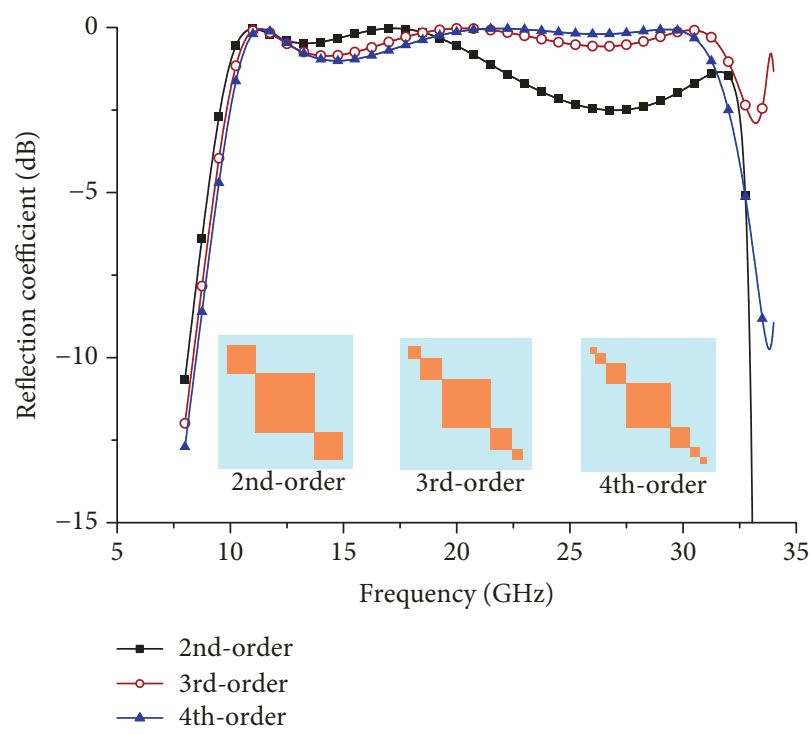

FIgURE 2: Discussion on the iteration of the QFS.

The proposed PCM is applied in the RCS reduction of a microstrip antenna. The validity in the RCS reduction is proved by both measurements and simulations.

\section{Design of PCM}

2.1. Design of the PCM Unit Cell. The geometry of the PCM unit is shown in Figure 1. The unit is composed of three layers: quadrate fractal structure (QFS) (top layer), metal plane (bottom layer), and substrate with $W=6 \mathrm{~mm}$ in the side length (intermediate layer). The QFS consists of three squares with side lengths $L_{1}=2.2 \mathrm{~mm}, L_{2}=1 \mathrm{~mm}$, and $L_{3}=0.5 \mathrm{~mm}$, respectively. Square patches are connected by a strip with $D=0.1 \mathrm{~mm}$ in width. The intermediate layer is a substrate with a relative permittivity of 2.62 and $H=2 \mathrm{~mm}$ in thickness.

All the simulations in this study are done with the Ansys HFSS software based on the finite element method (FEM). By 


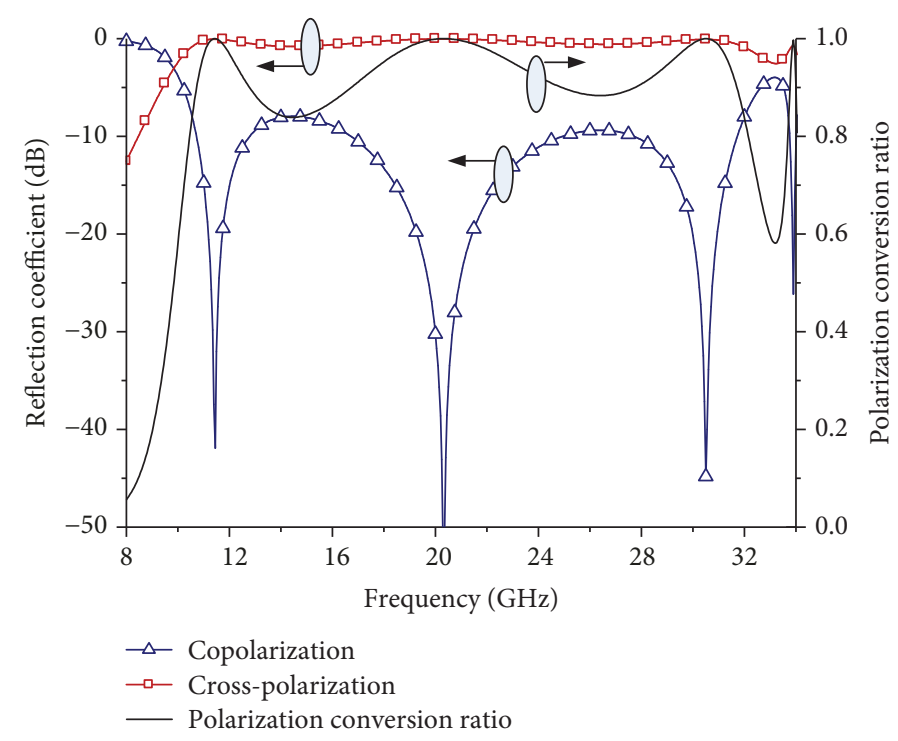

FIgURE 3: Reflection and polarization conversion performance of the PCM.

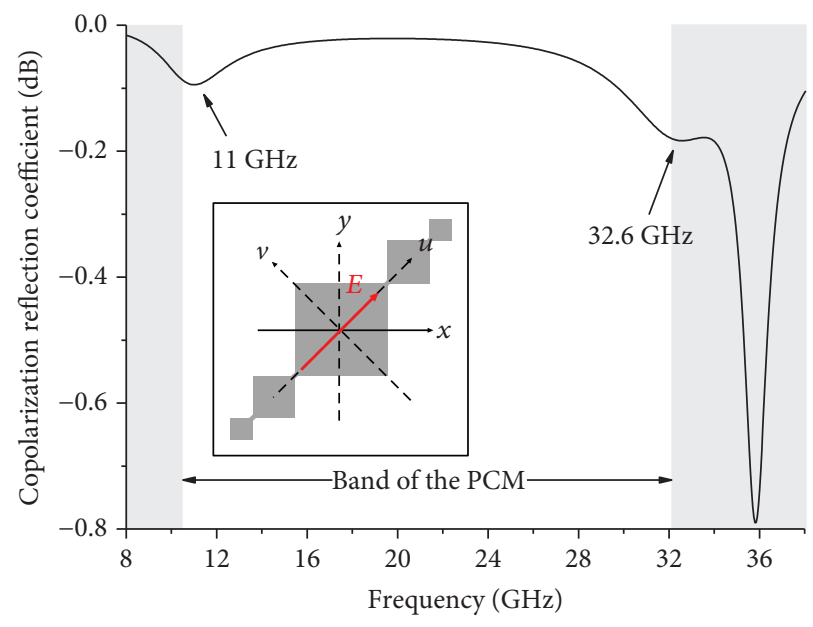

(a)

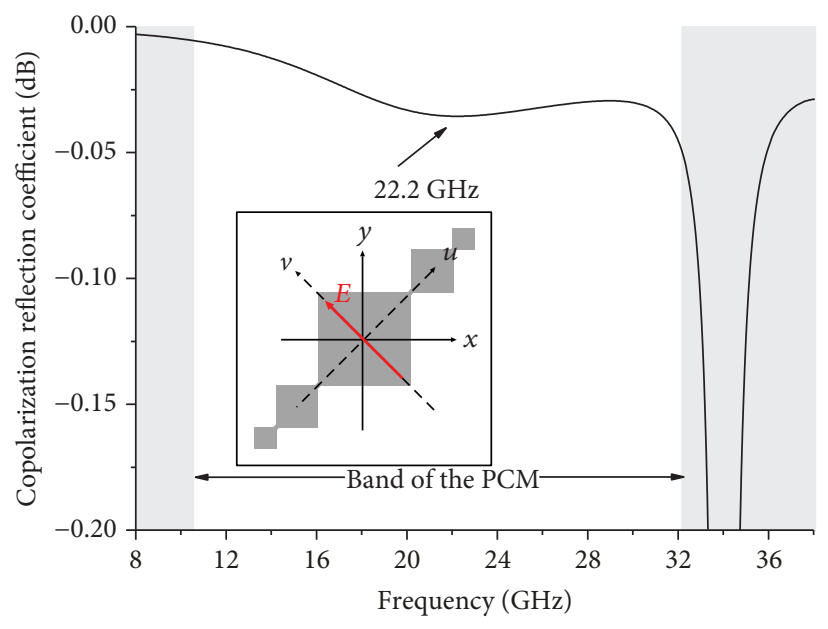

(b)

FIGURE 4: Copolarization reflection of $u$ - and $v$-polarized incidence: (a) $u$-polarized incidence; (b) $v$-polarized incidence.

arranging the units periodically on a plane, a PCM is obtained whose reflection and polarization conversion performances are studied. In order to find out the performances of the element in different iterations, the 2nd-order, 3rdorder, and 4th-order fractal elements are simulated as shown in Figure 2. From the reflection coefficient curves, it can be observed that the curve of the 2nd-order fractal structure is not smooth enough to provide a stable wideband PCR curve. In terms of the operating bandwidth and smoothness, the 3rd-order fractal structure is obviously a better choice than the 4th-order one.

Based on the discussion above, the 3rd-order QFS is used and the performances are shown in Figure 3 when the incident waves are $x$-polarized (the same as $y$-polarized due to the symmetric structure of QFS). According to the red and blue lines, the PCM keeps a high crosspolarized reflection coefficient when linear-polarized waves light the PCM normally. Under the condition of crosspolarization reflection coefficient great than $-1 \mathrm{~dB}$, the operating band of the PCM is from $10.45 \mathrm{GHz}$ to $32.15 \mathrm{GHz}$ (relative bandwidth of $101.9 \%$ ).

Furthermore, to exhibit the performances of the PCM more visually, polarization conversion ratio is also shown in Figure 3 by a black line. In the operating band of the PCM, the polarized conversion ratio is greater than $80 \%$ which means that $80 \%$ of the linear incident power is converted into the cross-polarized power. There are three peak values at $11 \mathrm{GHz}, 22.2 \mathrm{GHz}$, and $32.6 \mathrm{GHz}$ where the PCM can work with a polarization conversion ratio closing to $100 \%$.

To interpret the mechanism of the polarization conversion, the cases of $u$ - and $v$-polarization incidence are studied. As shown in Figure 4, three resonant frequency points within the operating band of the PCM can be found at $11 \mathrm{GHz}$, 


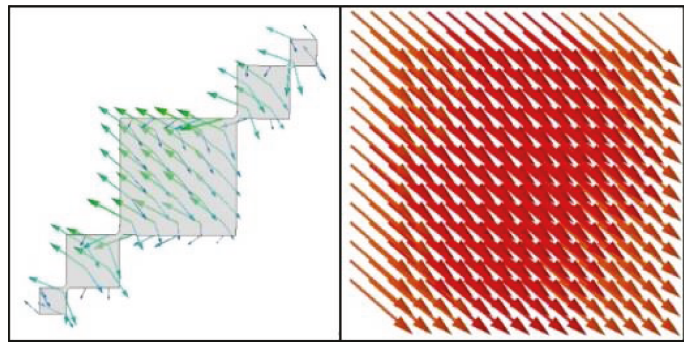

(a)

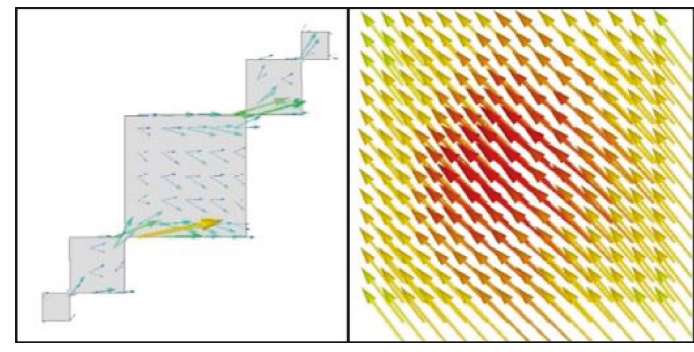

(b)

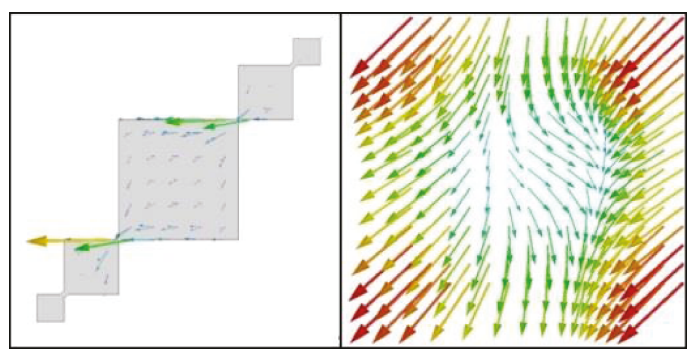

(c)

FIgURE 5: Current distribution on the QFS and ground plane at the resonant frequency points: (a) $11.1 \mathrm{GHz}$; (b) $22.2 \mathrm{GHz}$; (c) $32.6 \mathrm{GHz}$.

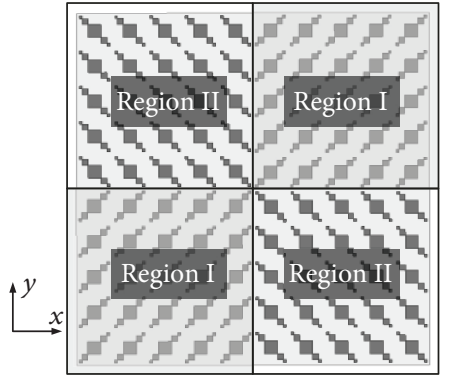

FIGURE 6: Configuration of the chessboard-like plane.

22.2 $\mathrm{GHz}$, and $32.6 \mathrm{GHz}$ which correspond with the three peak values of the polarization conversion ratio. The surface current distributions of the QFS and ground plane at $11 \mathrm{GHz}$, 22.2 GHz, and $32.6 \mathrm{GHz}$ are shown in Figure 5. The induced current generated by the QFS is opposite to that of the ground plane, which constitutes an equivalent magnetic resonator at $11 \mathrm{GHz}$. At $22.2 \mathrm{GHz}$, the direction of surface current for QFS is orthogonal to that of the ground plane, which leads to the orthogonal reflection of the electric field. When the frequency of the incident wave increases to $32.6 \mathrm{GHz}$, the induced current has the same direction as that of the ground plane, which constitutes an equivalent electric resonator.

2.2. Mechanism of RCS Reduction. As shown in Figure 6, the chessboard-like plane is divided into two regions: region I (upper right block and lower left block) and region II (upper left block and lower right block). In region I, two $5 \times 5$ arrays are formed by the QFS elements, while in region II, the other two $5 \times 5$ arrays are formed by the mirror elements of the QFS.

When the incident wave illuminates the chessboard-like plane normally, the reflected wave will be the superposition of the reflected waves from region I and region II. It is supposed that the reflected electric field from region $\mathrm{I}$ is

$$
\bar{E}_{1}=A_{1} \bullet e^{j \varphi_{1}}
$$

Due to the chessboard arrangement, the reflected wave from region II is in the same amplitude and $180^{\circ}$ phase difference with that from region I. The reflected electric field from region $\mathrm{II}$ is

$$
\bar{E}_{2}=A_{2} \bullet e^{j \varphi_{2}}=A_{1} \bullet e^{j\left(\varphi_{1} \pm 180^{\circ}\right)} .
$$

With $\bar{E}_{1}$ and $\bar{E}_{2}$, the total reflected electric field is

$$
\bar{E}_{\text {chessboard }}=\bar{E}_{1}+\bar{E}_{2}=A_{1} \bullet e^{j \varphi_{1}}+A_{1} \bullet e^{j\left(\varphi_{1} \pm 180^{\circ}\right)} .
$$

It can be found that the reflected wave will be too weak to be detected due to the superposition.

However, the phase difference between $\bar{E}_{1}$ and $\bar{E}_{2}$ is not exactly $180^{\circ}$ for most of the frequency points in the operating band. In this case, the total reflected electric field is

$$
\bar{E}_{\text {chessboard }}=A_{1} \bullet e^{j \varphi_{1}}\left(1+e^{j\left(\varphi_{2}-\varphi_{1}\right)}\right) .
$$

Moreover, in the metal plane, the total reflected electric field is

$$
\bar{E}_{\text {metal }}=2 A_{1} \cdot e^{j \varphi_{1}}
$$

In order to keep the reflected power in a low level, for example,

$$
\frac{\left|\bar{E}_{\text {chessboard }}\right|^{2}}{\left|\bar{E}_{\text {metal }}\right|^{2}} \leq-10 \mathrm{~dB},
$$




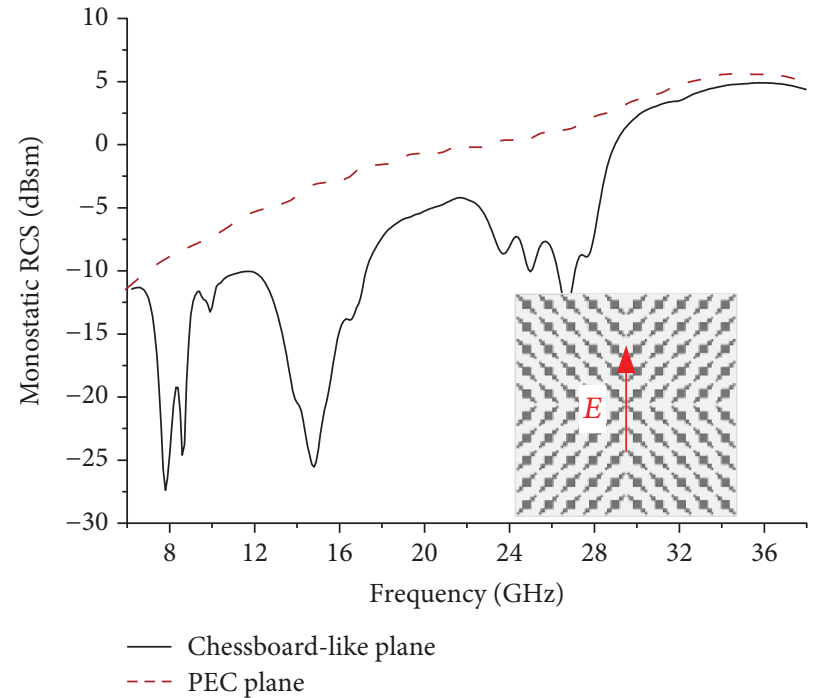

(a)

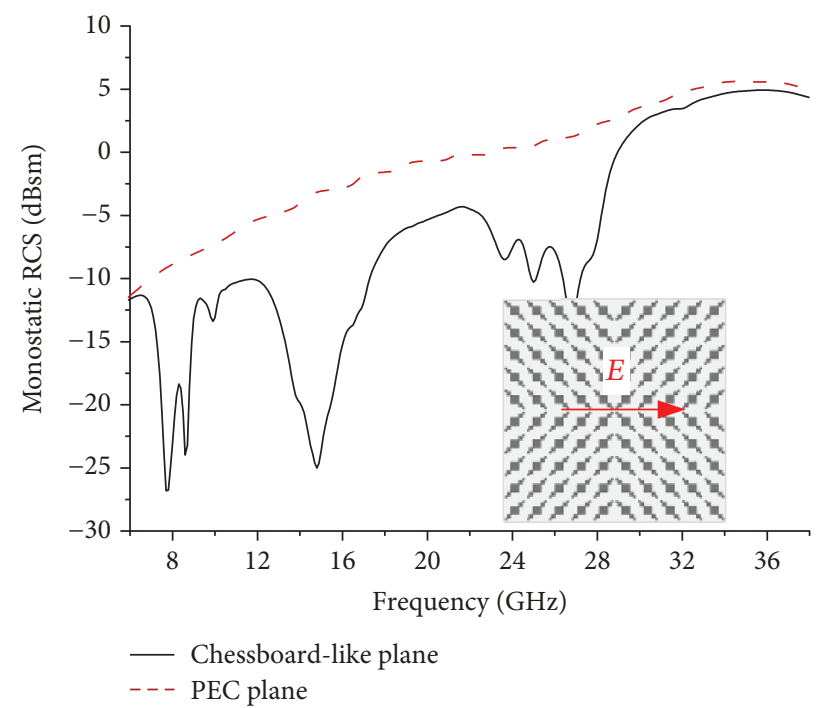

(b)

FIGURE 7: Monostatic RCS of the chessboard-like plane and PEC plane for normal incidence: (a) $x$-polarized incidence; (b) $y$-polarized incidence.

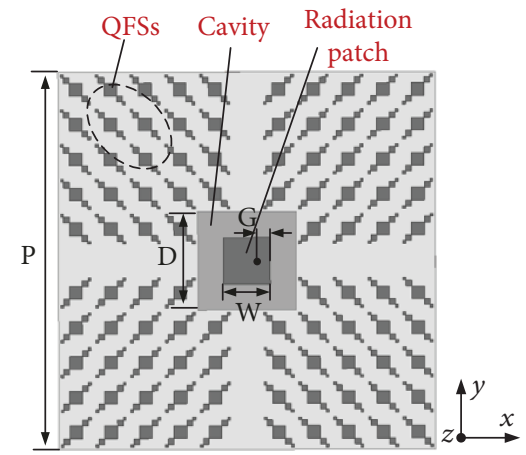

(a)

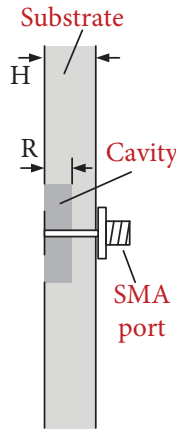

(b)

FIgURE 8: Configuration of the proposed antenna: (a) top view; (b) side view.

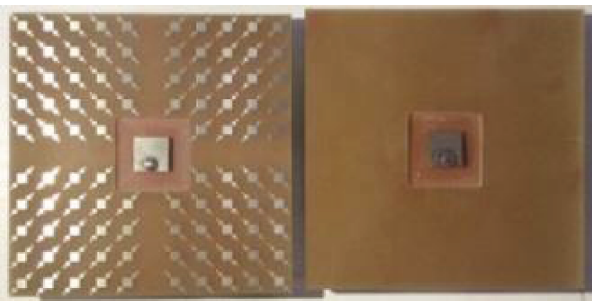

FIgURE 9: Prototypes of the proposed and reference antennas.

then

$$
\cos \left(\varphi_{2}-\varphi_{1}\right) \leq-0.8
$$

The phase difference will be

$$
143^{\circ} \leq\left|\varphi_{2}-\varphi_{1}\right| \leq 217^{\circ} \text {. }
$$

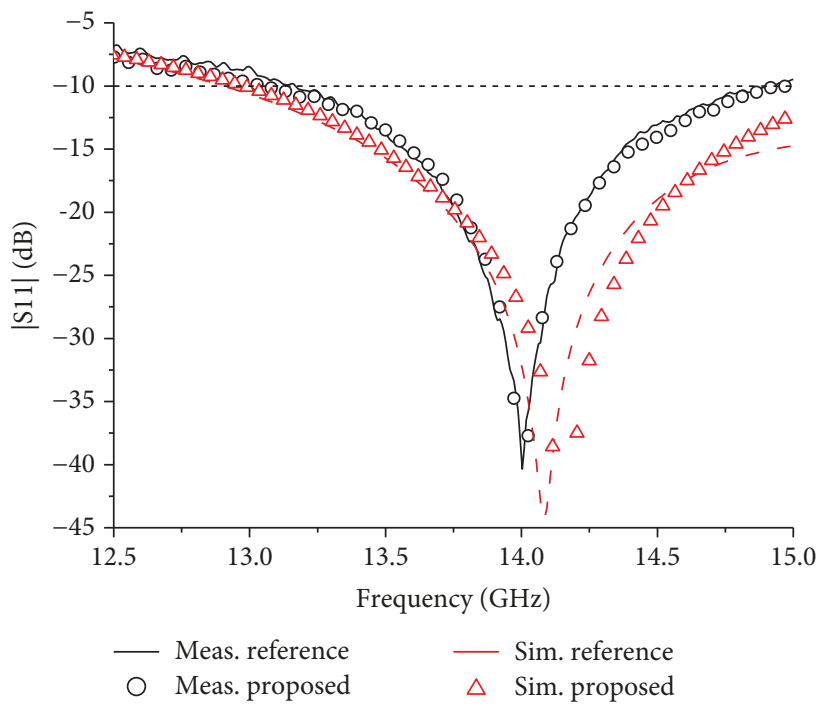

FIGURE 10: Measured and simulated $|\mathrm{S} 11|$ of the antennas. 


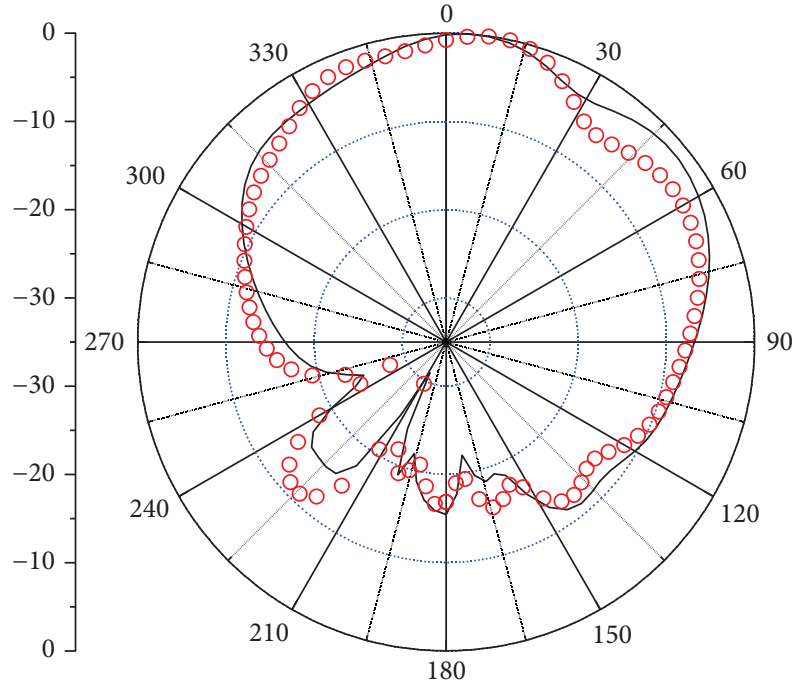

Proposed antenna
Reference antenna

(a)

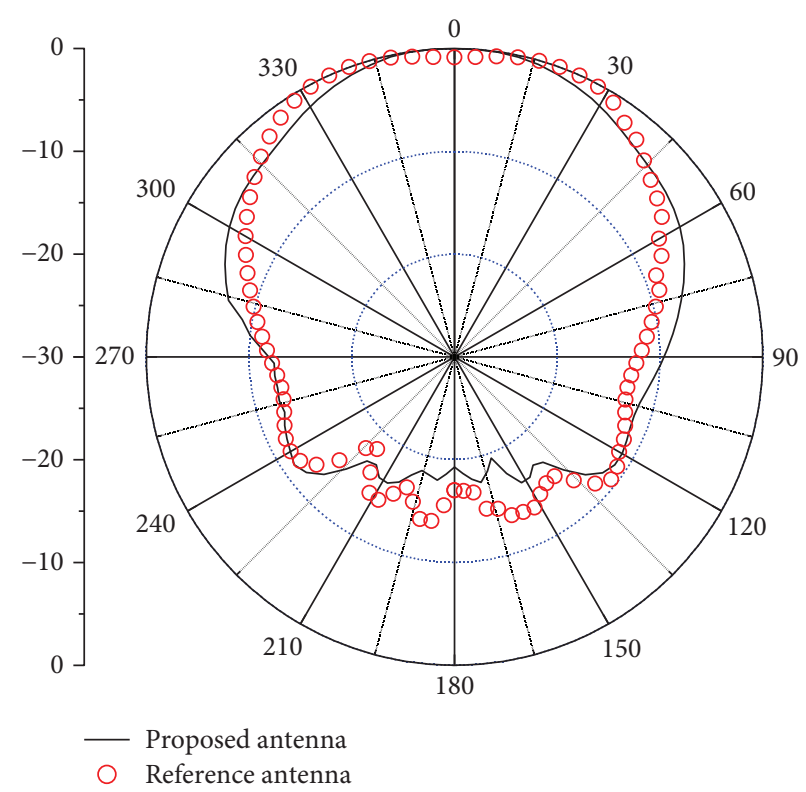

(b)

FIGURE 11: Normalized radiation patterns of the proposed and reference antenna at $14 \mathrm{GHz}$ : (a) $x z$-plane; (b) $y z$-plane.

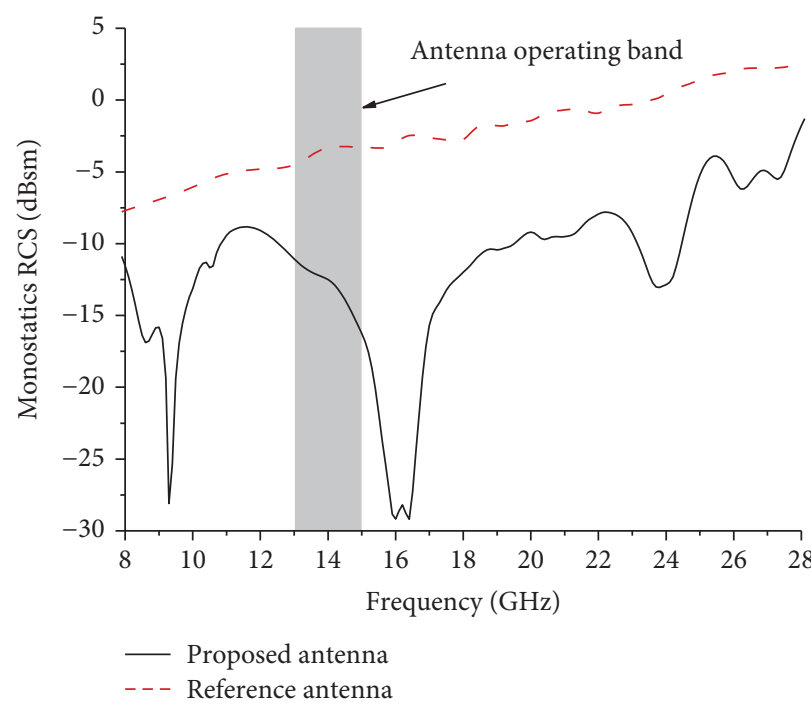

(a)

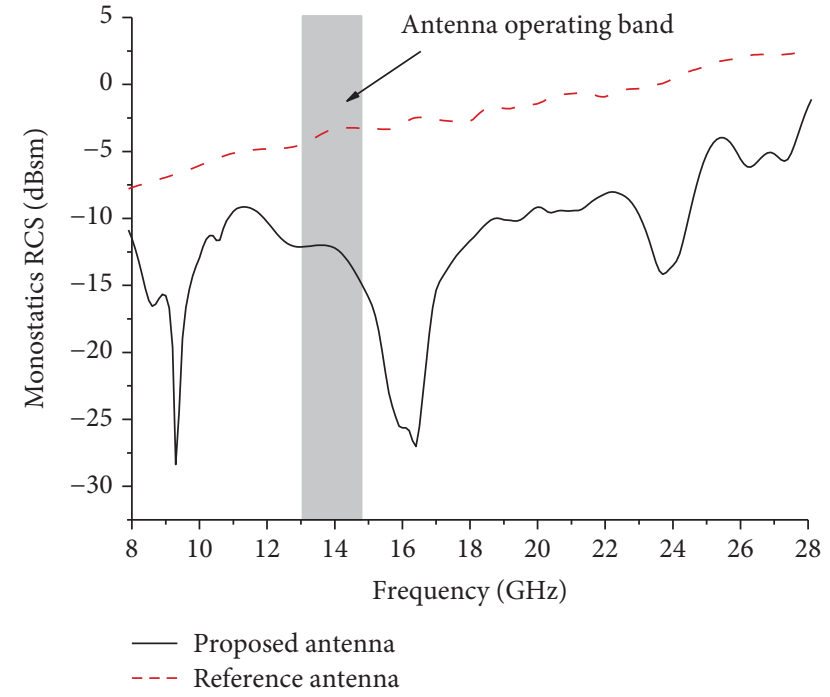

(b)

FIGURE 12: Monostatic RCS of the proposed and reference antennas for normal incidence: (a) $x$-polarized incidence; (b) $y$-polarized incidence.

From (8), the reflected power will be obviously reduced when the reflected phase difference between region I and region II is $180^{\circ} \pm 30^{\circ}$.

The monostatic RCS curves of the chessboard-like plane and perfect electric conductor (PEC) plane which are illuminated by the $x$-polarized and $y$-polarized incident waves are shown in Figure 7 . When the plane is illuminated normally, the structure of the plane is all the same for both $x$-polarized and $y$-polarized incident waves. Theoretically, the same resonant performances of the plane could be obtained due to the symmetrical structure of the chessboard-like plane. Therefore, the monostatic RCS curves of the proposed antenna are almost matching between the $x$-polarized and $y$ -polarized incident cases. It can be found that the reflected power is well suppressed from $6 \mathrm{GHz}$ to $38 \mathrm{GHz}$ with a relative bandwidth of $145.5 \%$ for both $x$ - and $y$-polarized incidence cases. From $7.2 \mathrm{GHz}$ to $28.7 \mathrm{GHz}$, a relative bandwidth of $119.7 \%$, the RCS of the chessboard-like plane is reduced for more than $4 \mathrm{dBsm}$.

\section{Antenna RCS Reduction Based on PCM}

The proposed PCM is applied in the RCS reduction of the microstrip antenna in this section. The configuration of the 
proposed antenna is shown in Figure 8. It consists of radiation patch, ground plane, and QFSs in chessboard arrangement. The QFSs and radiation patch are on the same side of the substrate with a relative permittivity of 2.62. Behind the radiation patch, a cavity is inserted into the substrate to improve the impendence matching of the antenna. The ground plane is on the other side of the substrate. The sizes of the proposed antenna are shown as follows: $P=65 \mathrm{~mm}, H=2 \mathrm{~mm}, R=1.2 \mathrm{~mm}, D=17 \mathrm{~mm}$, $W=8 \mathrm{~mm}$, and $G=2.2 \mathrm{~mm}$. As a reference, another antenna is designed without QFSs. The prototypes of the proposed and reference antennas are shown in Figure 9.

As shown in Figure 10, the $|S 11|$ curves of the proposed and reference antennas fit well which means that the antennas share the same operating band. Under the condition of $|\mathrm{S} 11| \leq-10 \mathrm{~dB}$, the measured operating bands of the proposed and reference antennas are from $13.04 \mathrm{GHz}$ to $14.97 \mathrm{GHz}$ (relative bandwidth of $13.8 \%$ ) and from $13.17 \mathrm{GHz}$ to $14.89 \mathrm{GHz}$ (relative bandwidth of $12.3 \%$ ), respectively. However, frequency shifts appear between the simulated and measured curves. The frequency shifts, which are prevalent in antenna engineering, are caused by the errors in the fabrication and the electrical parameters of the substrate.

Figure 11 is the normalized radiation patterns of the proposed and reference antennas at $14 \mathrm{GHz}$. The measurements are carried out in the anechoic chamber. The curves are in good agreement with the simulated ones. On the working direction $\left(\theta=0^{\circ}\right)$, the gains of the proposed and reference antennas are $7.13 \mathrm{dBi}$ and $6.2 \mathrm{dBi}$. The radiation patterns and gains show that the proposed antenna has similar radiation performances with the reference antenna. The mismatching between the curves is caused by the coupling between the PCM element and the antenna radiator.

The monostatic RCS of the proposed and reference antennas is studied when the incident plane waves illuminate the antennas normally. It can be found from Figure 12 that, with the radiation performance hold, the RCS of the proposed antenna is obviously lower than that of the reference antenna from $8 \mathrm{GHz}$ to $28 \mathrm{GHz}$ for both the $x$ - and $y$-polarized incidence. Especially in the operating band of the proposed antenna, the RCS is reduced by more than $6.8 \mathrm{~dB}$ for the $x$-polarized incidence and $7.7 \mathrm{~dB}$ for the $y$-polarized incidence.

\section{Conclusion}

Polarization conversion metasurface (PCM) with wide operating band and high polarization conversion ratio is proposed in this paper. The proposed PCM element is designed with a 3rd-order quadrate fractal structure. The polarization conversion ratio is greater than $80 \%$ from $10.45 \mathrm{GHz}$ to $32.15 \mathrm{GHz}$. The performances of the proposed PCM and antenna are studied by measurement and simulation. The results show that, with the radiation performance hold, the monostatic RCS the proposed antenna is obviously reduced from $8 \mathrm{GHz}$ to $28 \mathrm{GHz}$ which completely covers the operating band of the antenna.

\section{Data Availability}

The data used to support the findings of this study are available from the corresponding author upon request.

\section{Conflicts of Interest}

The authors declare that they have no conflicts of interest.

\section{Acknowledgments}

This work was supported by the National Key R\&D Program of China (2017YFF0205200).

\section{References}

[1] W. Chen, C. A. Balanis, and C. R. Birtcher, "Checkerboard EBG surfaces for wideband radar cross section reduction," IEEE Transactions on Antennas and Propagation, vol. 63, no. 6, pp. 2636-2645, 2015.

[2] W. Chen, C. A. Balanis, and C. R. Birtcher, "Dual wide-band checkerboard surfaces for radar cross section reduction," IEEE Transactions on Antennas and Propagation, vol. 64, no. 9, pp. 4133-4138, 2016.

[3] C. Yang, M. He, C. F. Zhang, and H. J. Sun, "Radar cross section reduction for microstrip antenna using broadband chessboard artificial magnetic conductor structure," in 2016 IEEE International Conference on Microwave and Millimeter Wave Technology (ICMMT), pp. 777-779, Beijing, China, June 2016.

[4] S. H. Esmaeli and S. H. Sedighy, "Wideband radar crosssection reduction by AMC," Electronics Letters, vol. 52, no. 1, pp. 70-71, 2016.

[5] Z. J. Han, W. Song, and X. Q. Sheng, "Gain enhancement and RCS reduction for patch antenna by using polarizationdependent EBG surface," IEEE Antennas and Wireless Propagation Letters, vol. 16, pp. 1631-1634, 2017.

[6] X. Kang, J. Su, H. Zhang, Z. Li, and Y. (. L.). Yang, "Ultra-wideband RCS reduction of microstrip antenna array by optimised multi-element metasurface," Electronics Letters, vol. 53, no. 8, pp. 520-522, 2017.

[7] Y. Jia, Y. Liu, Y. J. Guo, K. Li, and S.-X. Gong, "Broadband polarization rotation reflective surfaces and their applications to RCS reduction," IEEE Transactions on Antennas and Propagation, vol. 64, no. 1, pp. 179-188, 2016.

[8] Y. Liu, Y. Hao, K. Li, and S. Gong, "Radar cross section reduction of a microstrip antenna based on polarization conversion metamaterial," IEEE Antennas and Wireless Propagation Letters, vol. 15, pp. 80-83, 2016.

[9] Y. Liu, K. Li, Y. Jia, Y. Hao, S. Gong, and Y. J. Guo, "Wideband RCS reduction of a slot array antenna using polarization conversion metasurfaces," IEEE Transactions on Antennas and Propagation, vol. 64, no. 1, pp. 326-331, 2016.

[10] C. Kong, Q. Guo, Z. Li, and Z. Wu, "Wideband RCS reduction based on polarization conversion metasurface," in 2016 11th International Symposium on Antennas, Propagation and EM Theory (ISAPE), pp. 696-698, Guilin, China, October 2016.

[11] Y. Jia, Y. Liu, Y. J. Guo, K. Li, and S. Gong, "A dual-patch polarization rotation reflective surface and its application to ultra-wideband RCS reduction," IEEE Transactions on Antennas and Propagation, vol. 65, no. 6, pp. 3291-3295, 2017. 
[12] L. Zhang and T. Dong, "Low RCS and high-gain CP microstrip antenna using SA-MS," Electronics Letters, vol. 53, no. 6, pp. 375-376, 2017.

[13] Z. Wei, Y. Cao, Y. Fan, X. Yu, and H. Li, "Broadband polarization transformation via enhanced asymmetric transmission through arrays of twisted complementary split-ring resonators," Applied Physics Letters, vol. 99, no. 22, p. 221907, 2011.

[14] H. Shi, J. Li, A. Zhang, J. Wang, and Z. Xu, "Broadband cross polarization converter using plasmon hybridizations in a ring/disk cavity," Optics Express, vol. 22, no. 17, pp. 2097320981, 2014.

[15] H. Chen, H. Ma, S. Qu et al., "Ultra-wideband polarization conversion metasurfaces," in Proceedings of 2014 3rd Asia-Pacific Conference Antennas and Propagation, pp. 1009-1011, Harbin, China, July 2014.

[16] Y. Ji-Bao, M. Hua, W. Jia-Fu, F. Ming-De, L. Yong-Feng, and Q. Shao-Bo, "High-efficiency ultra-wideband polarization conversion metasurfaces based on split elliptical ring resonators," Acta Physica Sinica, vol. 64, no. 17, article 178101, 2015.

[17] L. Zhang, P. Zhou, H. Lu, H. Chen, J. Xie, and L. Deng, "Ultrathin reflective metamaterial polarization rotator based on multiple plasmon resonances," IEEE Antennas and Wireless Propagation Letters, vol. 14, pp. 1157-1160, 2015.

[18] X. Gao, X. Han, W. P. Cao, H. O. Li, H. F. Ma, and T. J. Cui, "Ultrawideband and high-efficiency linear polarization converter based on double V-shaped metasurface," IEEE Transactions on Antennas and Propagation, vol. 63, no. 8, pp. 3522-3530, 2015.

[19] Y. Liu, S. Xia, H. Shi, A. Zhang, and Z. Xu, "Dual-band and high-efficiency polarization converter based on metasurfaces at microwave frequencies," Applied Physics B, vol. 122, no. 6, p. 178, 2016. 


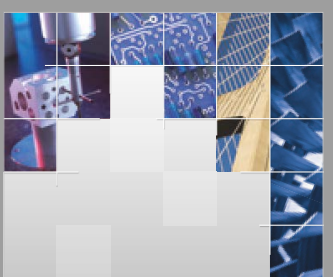

\section{Enfincering}
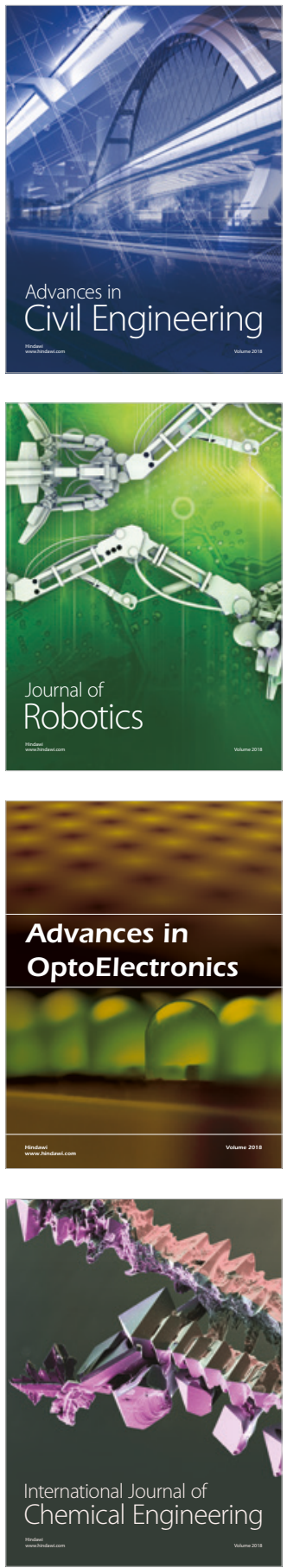

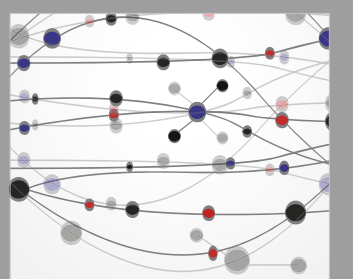

\section{Rotating \\ Machinery}

The Scientific World Journal

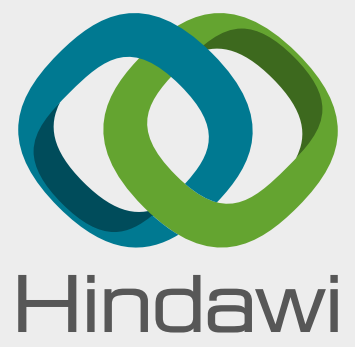

Submit your manuscripts at

www.hindawi.com
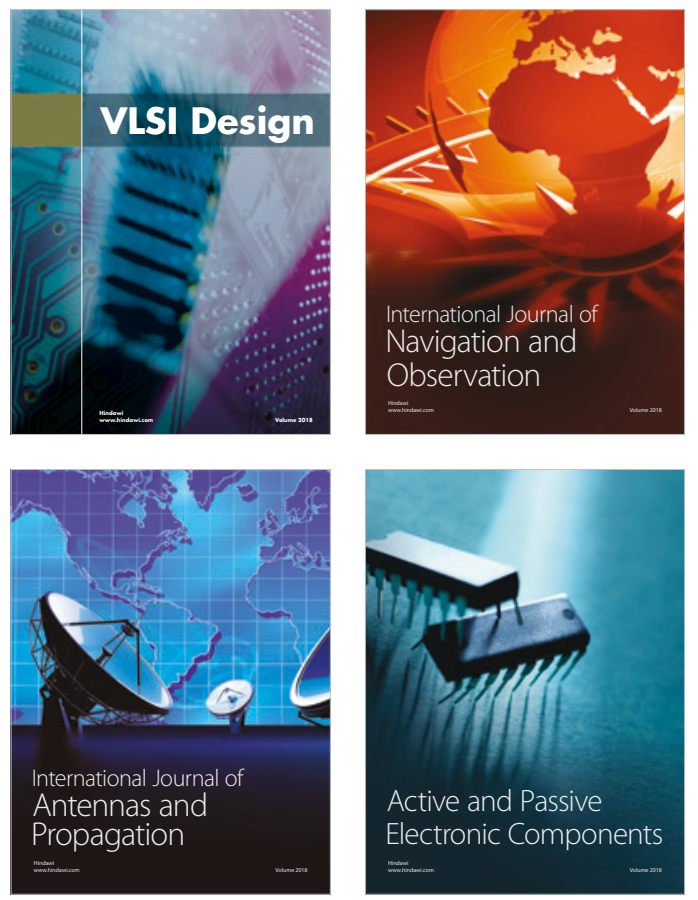
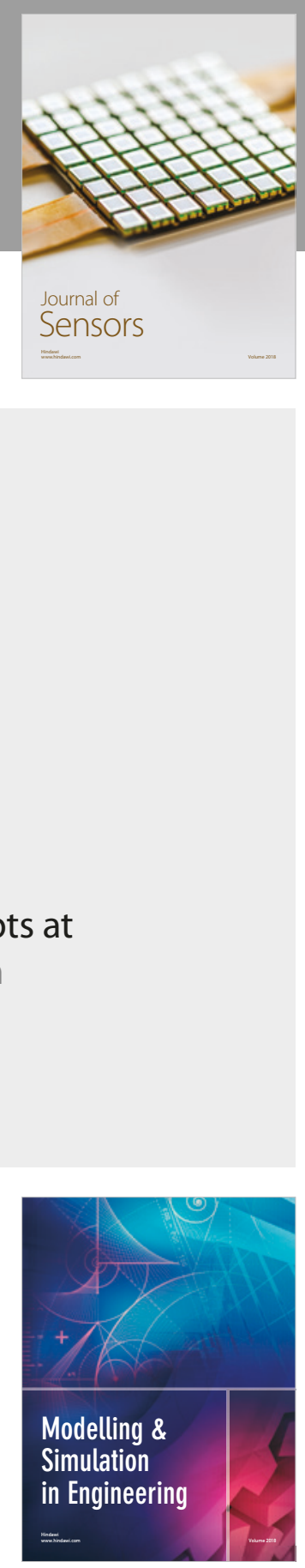

\section{Advances \\ Multimedia}
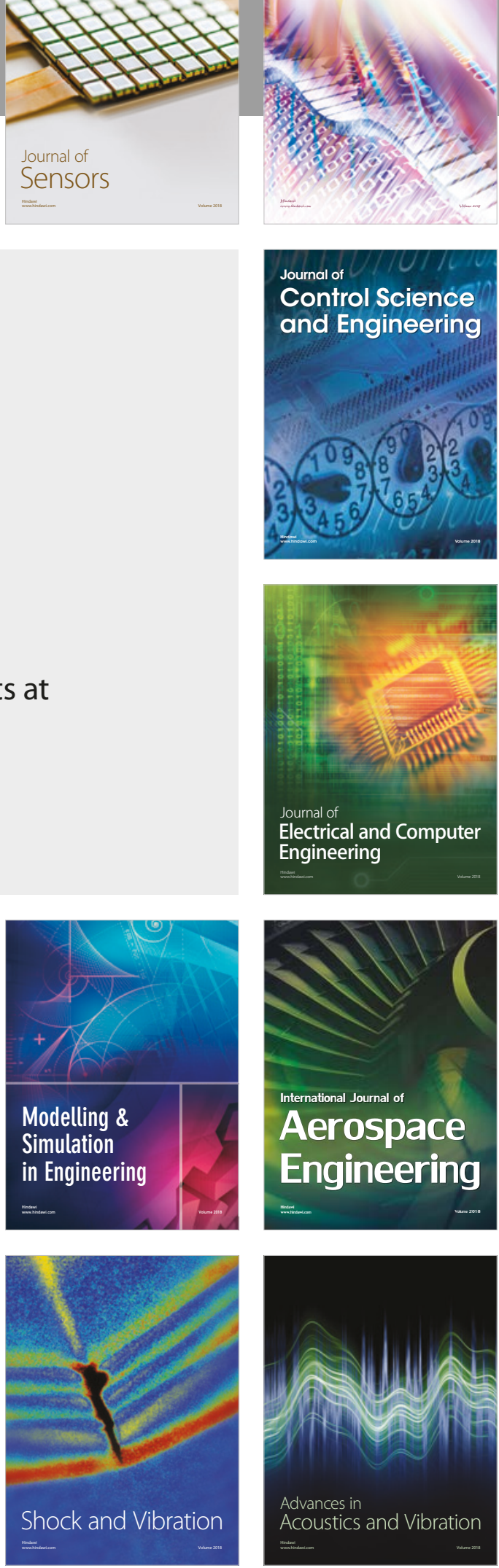